\title{
Impact of ohmic heating on MHD mixed convection flow of Casson fluid by considering Cross diffusion effect
}

https://doi.org/10.1515/nleng-2017-0144

Received July 8, 2017; revised June 15, 2018; accepted August 3, 2018.

Abstract: Present communication aims to discuss the impact of viscous dissipation on MHD flow, heat and mass transfer of Casson fluid over a plate by considering mixed convection. Nonlinear partial differential systems are reduced to the ordinary ones through transformation procedure. The modelled nonlinear systems are computed implementing RKF-45 scheme. Convergent solutions for velocity and temperature and concentration fields are given diagrammatically. The obtained results are compared with published literatures and reasonable agreement is found. It is found that, temperature profile increases by increasing values of Dufour parameter, whereas on opposite trend is observed in concentration profile for increasing values Soret parameter.

Keywords: Casson fluid, nonlinear thermal radiation, magnetic field, Cross diffusion effect, vertical surface, Joule heating, viscous dissipation

\section{Introduction}

Due to amicable applications of non-Newtonian fluid attracts many scientist and researchers. Casson fluid model is one of the non-Newtonian fluid model. Fluids exist in our background, viz., honey, soup, jelly, concentrated fruit juices, tomato sauce, etc., belong to Casson fluid model. Casson fluid is an example of shear thinning liquid. $\mathrm{Hu}$ -

B.J. Gireesha, N.G. Rudraswamy, Department of Studies and Research in Mathematics, Kuvempu University, Shankaraghatta-577 451, Shimoga, Karnataka, India

*Corresponding Author: K. Ganesh Kumar, Department of Mathematics, SJM Institution of technology, Chitradurga-577502, Karnataka, India, E-mail: ganikganesh@gmail.com

M.R. Krishnamurthy, Department of Mathematics, Jawaharlal Nehru National College of Engineering, Shimoga-577201, Karnataka, India S. Manjunatha, Department of Engineering Mathematics, Faculty of Engineering, Christ University, Mysore Road, Bengaluru-560074 India man blood is also an example of Casson fluid. The model of this fluid was first introduced by Casson [1] to anticipate the flow behavior of pigment-oil suspensions. Shehzad et al. [2] discussed the three dimensional MHD flow of Casson fluid in porous medium with heat generation. Khan et al. [3] investigated the comparative study of Casson fluid with homogeneous-heterogeneous reactions. Ramesh et al. [4] presented the radiation effect on hydromagnetic Casson fluid flow towards a stretched cylinder with suspension of liquid-particles. Seth et al. [5] investigated the hydromagnetic thin film flow of a Casson fluid in a nonDarcy porous medium with Joule dissipation. Keeping the aforesaid importance in mind, several researches have been reported considering this model (see [6-9]).

The viscous dissipation effect plays an important role in natural convection in various devices that are subjected to large deceleration, or which operate at high rotative speeds and also in strong gravitational field processes on large scales (on large planets) and geological process. Joule heating is also known as ohmic heating. It is a mechanism by which the passage of an electric current through a conductor produces heat. Joule heating has a variety of usage in industrial and technological processes such as electric stoves, electric heaters, incandescent light bulb, electric fuses, electronic cigarette, thermistor, food processing and several others. Chen [10] discussed the combined effects of Joule heating and viscous dissipation on magnetohydrodynamic flow past a permeable, stretching surface with free convection and radiative heat transfer. Chakraborty et al. [11] studied the thermal characteristics of EMHD flows in narrow channels with viscous dissipation and Joule heating under constant wall heat flux. Das et al. [12] presented the magnetohydrodynamic mixed convective slip flow over an inclined porous plate with viscous dissipation and Joule heating. Further recent investigations on Joule heating and viscous dissipation can be quoted through the studies [13-16].

In most of the above cited literature, linearized Rosseland approximation has been considered for radiation effect. This type of approximation involves the dimensionless parameters called Radiation parameter and Prandtl number which are sustainable if the temperature differ- 
ence between the plate and ambient fluid is small. But, for the larger temperature difference nonlinearized Rosseland approximation is valid. Mustafa et al. [17] discussed the influence of nonlinear thermal radiation in nanofluid flow over a vertical plate. Makinde and Animasaun [18] have employed homotopy analysis method to investigate the impact of nonlinear thermal radiation on magnetohydrodynamic bioconvection nanofluid flow. Some more recent studies on nonlinear thermal radiation effect for various fluids flow in distinct geometries can be seen in references [19-22].

The investigation regarding heat and mass transfer problems, the Soret and Dufour effects are ignored, on the basis that they are of a smaller order of magnitude than the effects described by Fourier's and Fick's laws. The heat flux induced by a concentration gradient is called Dufour effect or diffusion-thermo effect. These effects are considered as second order phenomena and are significant in areas such as hydrology, petrology, chemical reactors, drying processes, geosciences etc. Cheng [23] investigated the Soret and Dufour effects on free convection boundary layer over a vertical cylinder in a saturated porous medium. Bhattacharyya et al. [24] studied the Soret and Dufour effects on convective heat and mass transfer in stagnation point flow towards a shrinking surface. Seth et al. [25] analysed the Soret and Hall effects on unsteady MHD free convection flow of radiating and chemically reactive fluid past a moving vertical plate with ramped temperature in rotating system .Kataria and Patel [26] discussed the Soret and heat generation effects on MHD Casson fluid flow past an oscillating vertical plate embedded through porous medium. Seth et al. [27] discuss the hydromagnetic natural convection radiative flow of a viscoelastic nanofluid over a stretching sheet with Soret and Dufour effects. Seth et al. [28] investigated the natural convection flow in a non-Darcy medium with Soret and Dufour effects past an inclined stretching sheet. Kumar et al. [29] discuss the double diffusive MHD natural convection flow of Brinkman type nanofluid with diffusion thermo and chemical reaction. Kumar et al. [30] studied the cross diffusion effect on MHD mixed convection flow of nonlinear radiative heat and mass transfer of Casson fluid over a vertical plate.

In lookout of all the overhead mentioned applications, to the best of our perception no attention has been finalized for the study of MHD mixed convection flow of Casson fluid owing to stretching surface. Additionally, the properties of viscous dissipation, joule heating, Soret, Dufour effect and nonlinear radiation phenomena are incorporated. The graphical portrayal and tables are plotted and deliberated for the examination of varied somatic parameters by use of RKF-45 approach. Furthermore, the attained upshots are compared with former obtainable prose.

\section{Mathematical Formulation}

Consider a steady two dimensional laminar mixed convection flow of Casson liquid over a vertical stretching surface. The stretching velocity is assumed to be of the form $u_{w}(x)=b \sqrt{x}$ where $b$ is constant with $b>0$. A constant magnetic field of strength $B_{0}$ is applied in the $y$-direction, here an induced magnetic field is negligible compared with the applied field. This condition is usually well satisfied in terrestrial applications, especially so in (low-velocity) free convection flows. $T_{w}$ is the temperature of the sheet and $C_{w}$ is the concentration at the surface of the sheet. The ambient temperature and concentration represented by $T_{\infty}$ and $C_{\infty}$. The buoyancy forces arise due to the variations in temperature and concentration of fluid. The boussinesq approximation is invoked for the fluid properties to relate the density changes to temperature and concentration and to couple in this way the temperature and concentration fields to the flow field.

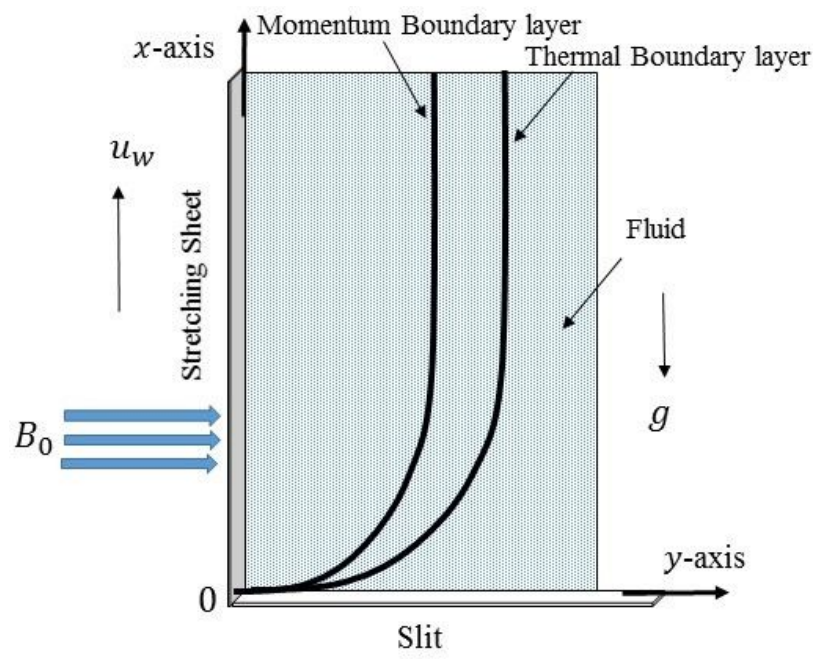

Fig. 1: Geometry of the problem.

Under these assumptions, the governing boundary layer equations can be expressed as,

$$
\frac{\partial u}{\partial x}+\frac{\partial v}{\partial y}=0
$$




$$
\begin{gathered}
u \frac{\partial u}{\partial x}+v \frac{\partial u}{\partial y}=v\left(1+\frac{1}{\beta}\right) \frac{\partial^{2} u}{\partial y^{2}} \\
+g \beta^{\star}\left(T-T_{\infty}\right)+g \beta^{\star \star}\left(C-C_{\infty}\right)-\frac{\sigma B_{0}^{2}}{\rho} u \\
u \frac{\partial T}{\partial x}+v \frac{\partial T}{\partial y}=\alpha \frac{\partial^{2} T}{\partial y^{2}}-\frac{1}{\rho c_{p}} \frac{\partial q_{r}}{\partial y}+\frac{D k_{T}}{c_{s} c_{p}} \frac{\partial^{2} C}{\partial y^{2}} \\
+\left(1+\frac{1}{\beta}\right) \frac{\mu}{\rho c_{p}}\left(\frac{\partial u}{\partial y}\right)^{2}+\frac{\sigma B^{2}}{c_{p} \rho} u^{2} \\
u \frac{\partial C}{\partial x}+v \frac{\partial C}{\partial y}=D \frac{\partial^{2} C}{\partial y^{2}}+\frac{D k_{T}}{T_{m}} \frac{\partial^{2} T}{\partial y^{2}}
\end{gathered}
$$

along with the relevant boundary conditions,

$$
\begin{aligned}
& u=u_{w}, v=0 \quad T=T_{w}, \quad C=C_{w} \text { at } y=0, u=0, \\
& T \rightarrow T_{\infty}, C \rightarrow C_{\infty} \text { as } y \rightarrow \infty .
\end{aligned}
$$

Using the Rosseland approximation for radiation, radiation heat flux $q_{r}$ is simplified as,

$$
q_{r}=-\frac{16 \sigma^{\star}}{3 k^{\star}} T^{3} \frac{d T}{d y}
$$

where $\sigma^{\star}$ and $k^{\star}$ are the Stefan-Boltzmann constant and the mean absorption coefficient respectively.

In view to (6), equation (3) reduces to

$u \frac{\partial T}{\partial x}+v \frac{\partial T}{\partial y}=\alpha\left(\frac{\partial^{2} T}{\partial y^{2}}\right)+\frac{16 \sigma^{\star}}{3 \rho c_{p} k^{\star}}\left[T^{3} \frac{\partial^{2} T}{\partial y^{2}}+3 T^{2}\left(\frac{\partial T}{\partial y}\right)^{2}\right]$

$+\frac{D k_{T}}{c_{s} c_{p}} \frac{\partial^{2} C}{\partial y^{2}}+\left(1+\frac{1}{\beta}\right) \frac{\mu}{\rho c_{p}}\left(\frac{\partial u}{\partial y}\right)^{2}+\frac{\sigma B_{0}^{2}}{c_{p} \rho} u^{2}$.

The following similarity transformations are introduced to transform the partial differential equations (1-4) to ordinary ones,

$$
\begin{aligned}
& \psi=\left(v x u_{w}(x)\right)^{\frac{1}{2}} f(\eta), \quad T=T_{\infty}\left(1+\left(\theta_{w}-1\right) \theta\right), \\
& \phi(\eta)=\frac{C-C_{\infty}}{C_{w}-C_{\infty}}, \quad \eta=\left(\frac{u_{w}(x)}{v x}\right)^{\frac{1}{2}} y,
\end{aligned}
$$

where $\theta_{w}=\frac{T_{w}}{T_{\infty}}$ is the temperature ratio parameter and $\psi$ is the stream function and is defined in the usual form as,

$$
u=\frac{\partial \psi}{\partial y} \text { and } v=-\frac{\partial \psi}{\partial x} \text {. }
$$
lows,

Reduced ordinary differential equations are as fol-

$$
\begin{aligned}
& \left(1+\frac{1}{\beta}\right) f^{\prime \prime \prime}+\frac{3}{4} f^{\prime \prime} f-\frac{1}{2} f^{\prime 2}+\lambda(\theta+N \phi)-M f^{\prime}=0, \\
& \theta^{\prime \prime}+R\left[\left(1+\left(\theta_{w}-1\right) \theta\right)^{3} \theta^{\prime \prime}+3\left(\theta_{w}-1\right) \theta^{\prime 2}\left(1+\left(\theta_{w}-1\right) \theta\right)^{2}\right] \\
& +\operatorname{Pr} \frac{3}{4} \theta^{\prime} f+\operatorname{PrD}_{f} \phi^{\prime \prime}+E c M f^{\prime 2}+\left(1+\frac{1}{\beta}\right) E c f^{\prime \prime 2}=0,
\end{aligned}
$$

$$
\frac{\phi^{\prime \prime}}{S c}+\frac{3}{4} f \phi^{\prime}+S r \theta^{\prime \prime}=0 .
$$

The transformed boundary conditions becomes,

$$
\begin{aligned}
f^{\prime}(\eta) & =1, \quad f(\eta)=0, \quad \theta(\eta)=1, \phi(\eta)=1 \\
\text { at } \eta & =0, f^{\prime}(\eta)=0
\end{aligned}
$$

Here $\lambda$ is the mixed convection parameter and $N$ is the buoyancy force parameter, which are given by $\lambda=$ $\frac{G r_{x}}{R e_{x}^{2}}, \quad N=\frac{G r_{x}^{*}}{G r_{x}}$ with $G r_{x}=\frac{g \beta^{*}\left(T_{w}-T_{\infty}\right) x^{3}}{v^{2}}$ is the thermal Grashof number, $G r_{x}^{\star}=\frac{g \beta^{\star \star}\left(C_{w}-C_{\infty}\right) x^{3}}{v^{2}}$ is the solutal Grashof number, $R e_{x}=\frac{u_{w}(x) x}{v}$ is the local Reynolds number, $M=$ $\frac{\sigma B_{0}^{2}}{\rho b}$ is the magnetic parameter, $R=\frac{16 \sigma^{\star} T_{\infty}^{3}}{3 k^{\star} k}$ is the radiation parameter, $\beta$ is the Casson parameter, $\operatorname{Pr}=\frac{v}{\alpha}$ is the Prandtl number, $S c=\frac{v}{D}$ is the Schmidt number, $S r=\frac{D k_{T}\left(T_{w}-T_{\infty}\right)}{T_{m} v\left(C_{w}-C_{\infty}\right)}$ is the Soret number, $D_{f}=\frac{D k_{T}\left(C_{w}-C_{\infty}\right)}{c_{s} c_{p} v\left(T_{w}-T_{\infty}\right)}$ is the Dufour number and $E c=\frac{u_{w}^{2}}{c_{p}\left(T_{w}-T_{\infty}\right)}$ is Eckert number,

The physical quantities of interest are the skin friction coefficient, local Nusselt number and the Sherwood number and are defined by,

$$
c_{f}=\frac{\tau_{w}}{\rho u_{w}^{2}}, \quad N u_{x}=\frac{u_{w} q_{w}}{k b\left(T_{w}-T_{\infty}\right)}, \quad S h_{x}=\frac{u_{w} j_{w}}{k b\left(C_{w}-C_{\infty}\right)},
$$

where $\tau_{w}, q_{w}$ and $j_{w}$ are the surface shear stress, heat flux and mass flux respectively andare given by;

$$
\tau_{w}=\mu\left(1+\frac{1}{\beta}\right)\left(\frac{\partial u}{\partial y}\right)_{y=0}, \quad q_{w}=-k\left(\frac{\partial T}{\partial y}+\left(q_{r}\right)_{w}\right)_{y=0}
$$

and

$$
j_{w}=-D_{m}\left(\frac{\partial c}{\partial y}\right)_{y=0} .
$$

Using similarity transformations we get;

$$
\begin{aligned}
& C_{f}\left(R e_{x}\right)^{\frac{1}{2}}=\left(1+\frac{1}{\beta}\right) f^{\prime \prime}(0), \\
& N u_{x}\left(R e_{x}\right)^{-\frac{1}{2}}=-\left[1+R \theta_{w}^{3}\right] \theta^{\prime}(0)
\end{aligned}
$$

and $\operatorname{Sh}\left(R e_{X}\right)^{-\frac{1}{2}}=-\phi^{\prime}(0)$.

\section{Numerical method}

The system of coupled extremely nonlinear ordinary differential equations (9)-(11) subject to the boundary conditions (12) are resolved numerically using RKF 45 technique. This method has been successfully used by the present author to resolve numerous problem associated with boundary layer flow and heat and mass transfer. During this method, the edge of the boundary layer has been chosen 
as $\eta=6$ i.e. $\eta \rightarrow \infty$ replaced by $\eta=6$ that is sufficient to realize the far field boundary conditions for all values of the parameters considered. To verify the validity and accuracy of our present method, we have shown a comparison of our results for the values of the temperature gradient with those reported by Chen [31].

\section{Result and discussions}

In the present-day interruption, we will scrutinize the properties of assorted parameters on the nonlinear radiative flow of Casson fluid with the impact of viscous dissipation and Joule heating. Additionally, the effects of MHD, Soret and Dufour effect are incorporated. The inducement of countless somatic parameters on velocity, temperature and concentration profiles are plotted and conferred. Therefore, for such objective, Figures 2 to 12 has been plotted. In the present problem we have considered the non-dimensional parameter values for numerical results as $M=0.5, N=0.1, \theta_{w}=1.2, \lambda=1, P r=5, \beta=$ 3, $R=0.5, S c=4, S r=0.7, D f=0.5$ and $E c=0.5$

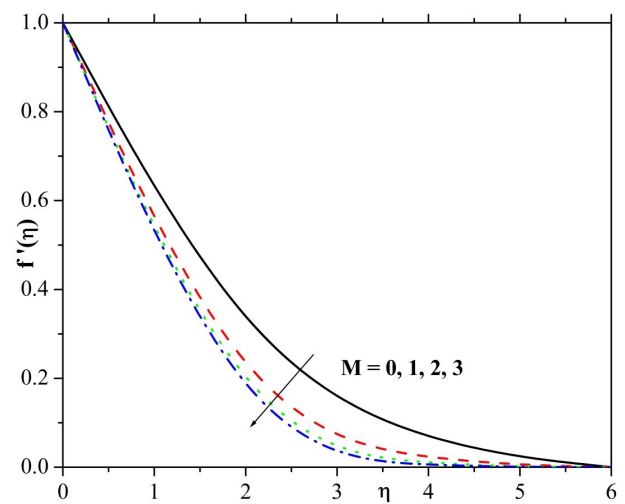

Fig. 2: Influence of $M$ on $f^{\prime}(\eta)$.

Figure 2 describes the effects of magnetic parameter $(M)$ on dimensionless velocity profile. In outlook of these plots we can detect that the velocity profile decreases with an enhancing values of $M$. From a physical point of view Lorentz force is positive and consequently as $M$ increases. This Lorentz force is also increase and hence expedites the flow. Figure 3 explicates the impact of Casson parameter $(\beta)$ for dimensionless velocity profile. It is revealed from this figure that, the velocity profile reduces with an en-

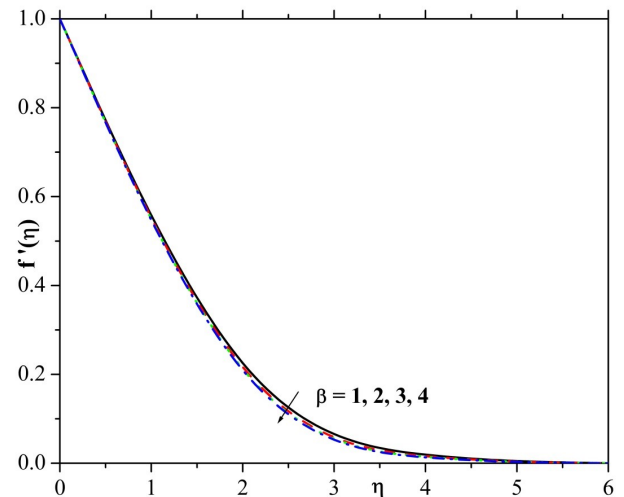

Fig. 3: Influence of $\beta$ on $f^{\prime}(\eta)$.

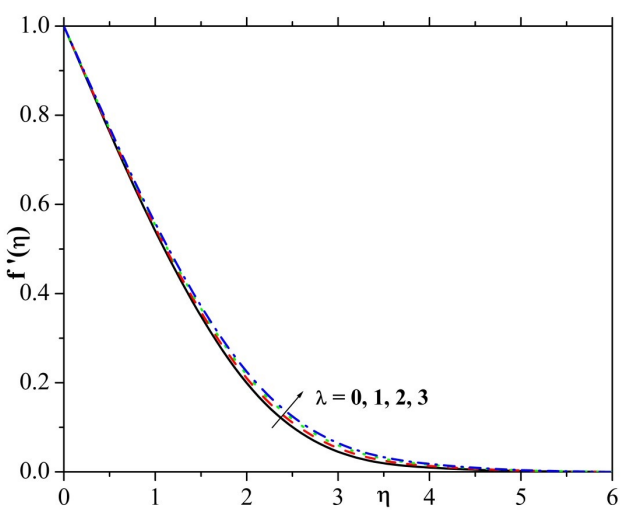

Fig. 4: Influence of $\lambda$ on $f^{\prime}(\eta)$

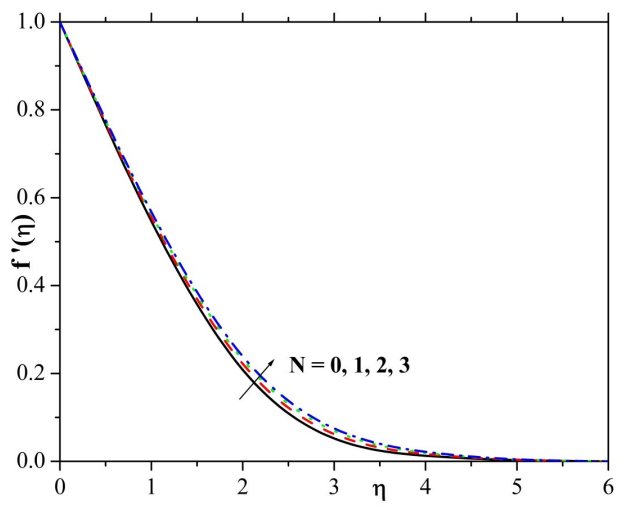

Fig. 5: Influence of $N$ on $f^{\prime}(\eta)$. 


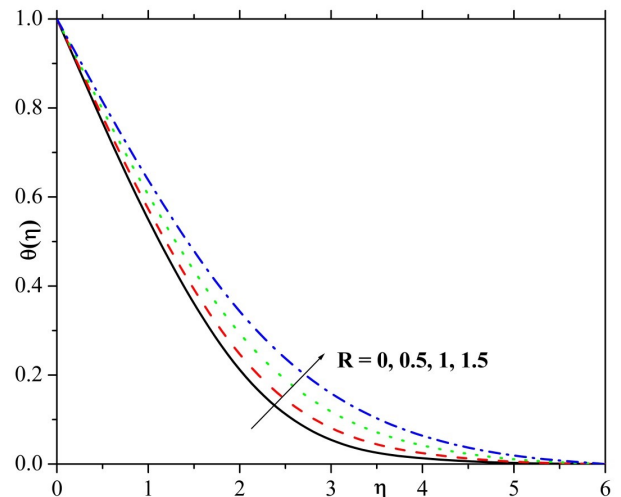

Fig. 6: Influence of $R$ on $\theta(\eta)$.

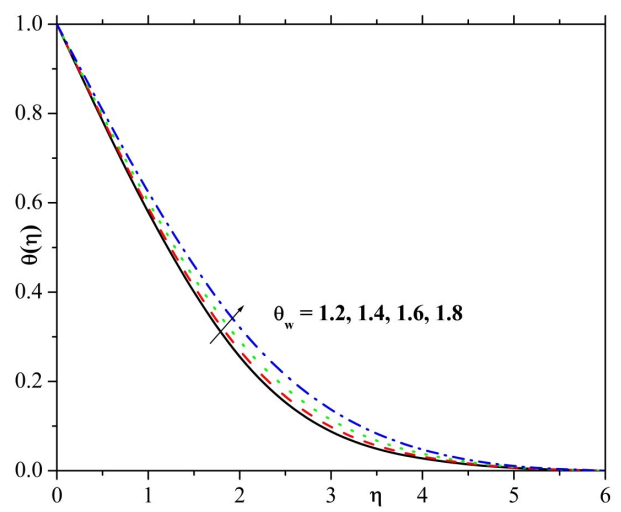

Fig. 7: Influence of $\theta_{w}$ on $\theta(\eta)$.

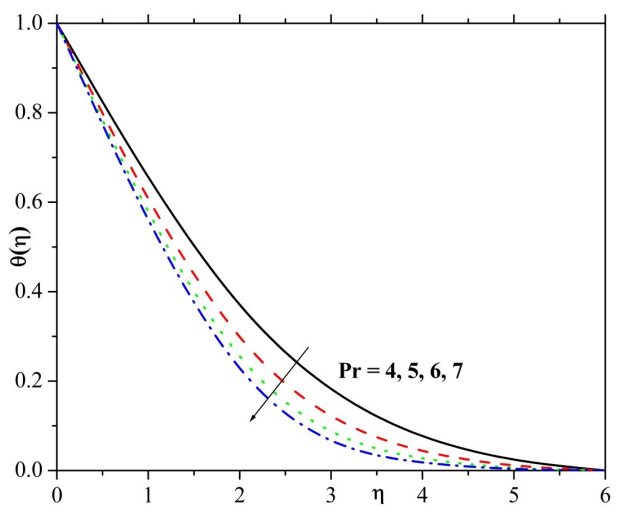

Fig. 8: Influence of $\operatorname{Pr}$ on $\theta(\eta)$.

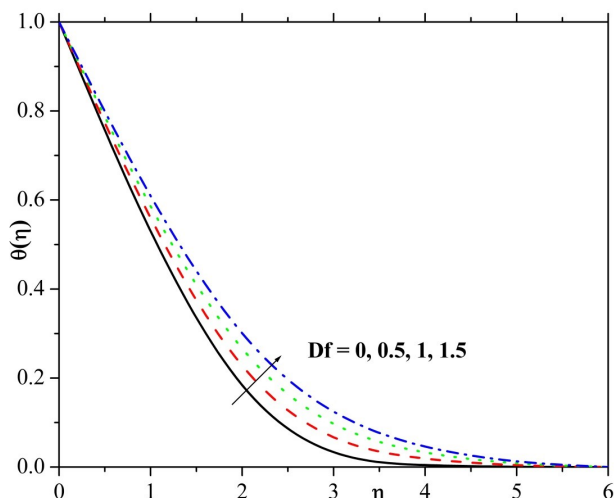

Fig. 9: Influence of $D f$ on $\theta(\eta)$.

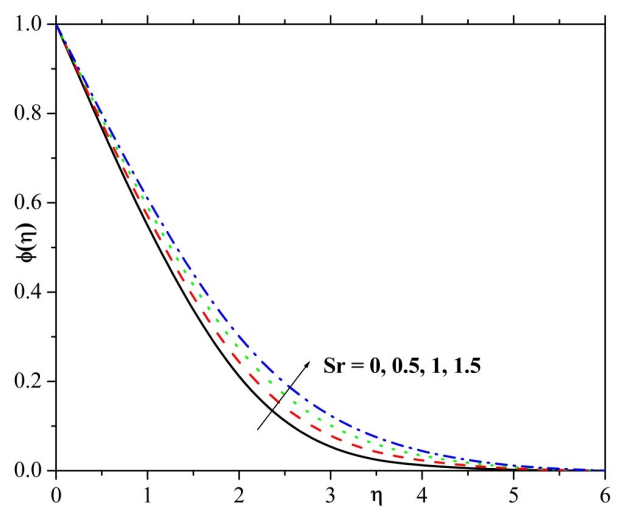

Fig. 10: Influence of $S r$ on $\phi(\eta)$.

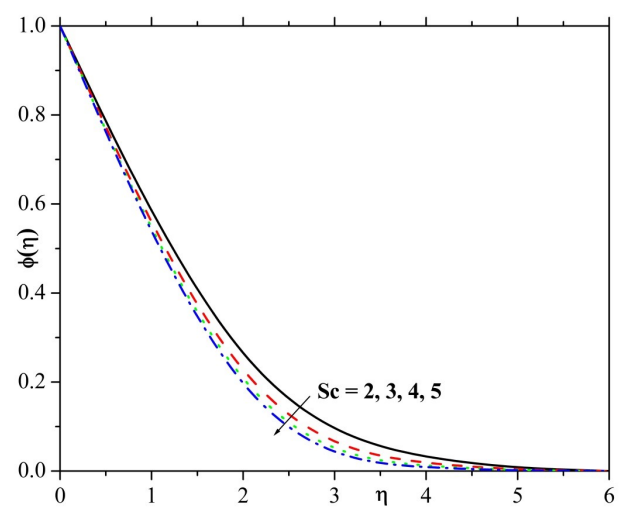

Fig. 11: Influence of $S c$ on $\phi(\eta)$. 


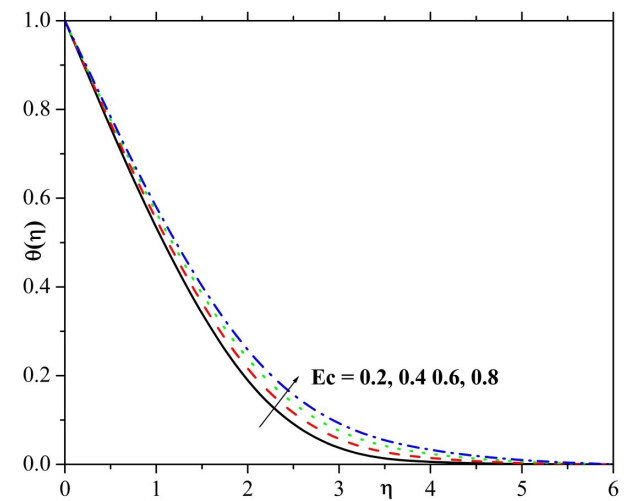

Fig. 12: Influence of $E c$ on $\theta(\eta)$.

hancing values of $\beta$. Physically, the higher values of Casson parameter reduce the yield stress in the fluid.

Figure 4 represents the behaviour of mixed convection parameter $(\lambda)$ on velocity distribution. It is observed that, the momentum boundary layer thickness increases for the higher values of $\lambda$. It is worth mentioning that $\lambda=0$ and $\lambda=0$ corresponds to the absence and presence of mixed convection parameter respectively. Also $\lambda>0$ indicates that heat is convected from the surface to the fluid flow i.e. cooling of the surface or heating the fluid. Therefore, velocity of the fluid increases. The impact of buoyancy force parameter $(N)$ for dimensionless velocity profile is presented in Figure 5. It is clear that, the velocity profile and its associated boundary layer thickness increases by increasing values of $N$.

Figure 6 deliberates the influence of radiation parameter $(R)$ on temperature distribution. From this figure one cane inform that, the temperature profile and its corresponding boundary layer thickness raises for increment values of $R$. Generally, higher value of $R$ produces additional heat to operating fluid that shows associate enhancement within the temperature field. Figure 7 examines the influence of temperature ratio parameter $\left(\theta_{w}\right)$ on temperature distribution. It is validated the fact that, an increasing values of $\theta_{w}$ improve the flow of the temperature profiles. This may happen because of increasing thermal conductivity of the flow.

Figure 9 illustrates the changes that are seen in temperature profiles due to increase in the values of Prandtl number. It is clear from figure that, the temperature profile decays for enrich values of $\mathrm{Pr}$, correspondingly thermal boundary layer thickness also reduces. This is because, Prandtl number related to the momentum diffusion and thermal diffusion in the bounder layer regime. The thermal boundary layer in the temperature distribution reduces by enhancing the values of Prandtl number. Hence, Prandtl number can be used to increase the speed of cooling in conducting flows.

Figure 9 characterizes the effect of Dufour parameter $(D f)$ on dimensionless temperature. It is clear from the graphical behaviour that, temperature profile and associated boundary layer thickness rises rapidly for growing values of $D f$. Figure 10 displays that the concentration distribution for escalating values of Soret parameter ( $S r)$. It is graphically displayed that the concentration profile is an increasing function of Soret parameter. Further, the solutal boundary layer thickness enhances for escalating values of Soret parameter.

Figure 11 is sketched to exhibit the dependence of the Schmidt number $(S c)$ on concentration profile. One can see from this figure that, the higher values of $S c$ decays the concentration profile and its corresponding boundary layer thickness. Physically, $S c$ is the ratio of momentum diffusivity and mass diffusivity. Mass diffusivity increases with increase in $S c$, consequently solutal boundary layer thickness decreases. The variation of the temperature profile for growing values of Eckert number $(E c)$ is graphically portrayed in Figure 12. It is graphically displayed that the temperature profile is an increasing function of $E c$. Further one can see that, the solutal boundary layer thickness increases by enhancing values of $E c$.

For the physical importance of the problem, the skinfriction coefficient, local Nusselt number and local Sherwood number are tabulated against different parameter. This is shows in table 1. From this table it is noted that increasing values of $D_{f}, P r, S c, \theta_{w}$ and $\beta$ is to decreases the Sherwood number and increase the Nusselt number. The opposite effect can be found when increase the parameters $M$ and $S r$.

\section{Conclusion}

In the present paper, numerical computations are performed for an impact of Joule heating on MHD mixed convection flow of Casson fluid by considering cross diffusion effect. Moreover, the effects of nonlinear thermal radiation and viscous dissipation are also employed. The concluding remarks embodying present work are listed as:

- $\quad$ Larger values of $\lambda$ and $N$ enrich the velocity profile and its correspondence boundary layer thickness.

- Higher values of $R$ and $\theta_{w}$ increases the rate of heat transfer as well as thermal boundary layer thickness. 
Table 1: Numerical values of $\left(1+\frac{1}{\beta}\right) f^{\prime \prime}(0), \phi^{\prime}(0)$ and $\left(1+R \theta_{w}^{3}\right) \theta^{\prime}(0)$ for various physical parameter.

\begin{tabular}{|c|c|c|c|c|c|c|c|c|c|c|c|c|c|}
\hline$D f$ & $\lambda$ & $N$ & $M$ & $P r$ & $R$ & $S c$ & $\mathrm{Sr}$ & $\theta_{w}$ & $\beta$ & $E c$ & $-\left(1+\frac{1}{\beta}\right) f^{\prime \prime}(0)$ & $-\phi^{\prime}(0)$ & $\begin{array}{l}-(1 \\
\left.+R \theta_{w}^{3}\right) \theta^{\prime}(0)\end{array}$ \\
\hline 0 & & & & & & & & & & & 1.76047 & 1.09700 & 1.24213 \\
\hline 0.5 & & & & & & & & & & & 1.76047 & 0.95292 & 1.32779 \\
\hline 1 & & & & & & & & & & & 1.76047 & 0.77498 & 1.43749 \\
\hline & 0 & & & & & & & & & & 1.59453 & 0.86594 & 1.57346 \\
\hline & 1 & & & & & & & & & & 1.36029 & 0.89537 & 1.58167 \\
\hline & 2 & & & & & & & & & & 1.14403 & 0.91985 & 1.58821 \\
\hline & & 1 & & & & & & & & & 1.76047 & 0.89023 & 1.58027 \\
\hline & & 2 & & & & & & & & & 1.60475 & 0.93619 & 1.59241 \\
\hline & & 3 & & & & & & & & & 1.57272 & 0.97357 & 1.60168 \\
\hline & & & 0.5 & & & & & & & & 1.17408 & 1.04326 & 1.50543 \\
\hline & & & 1 & & & & & & & & 1.49503 & 0.99327 & 1.40690 \\
\hline & & & 1.5 & & & & & & & & 1.76047 & 0.95292 & 1.32779 \\
\hline & & & & 4 & & & & & & & 1.76047 & 0.99718 & 1.01395 \\
\hline & & & & 5 & & & & & & & 1.76047 & 0.95292 & 1.32779 \\
\hline & & & & 6 & & & & & & & 1.76047 & 0.91178 & 1.61143 \\
\hline & & & & & 0 & & & & & & 1.76047 & 0.80275 & 0.95173 \\
\hline & & & & & 0.5 & & & & & & 1.76047 & 0.94057 & 1.27674 \\
\hline & & & & & 1 & & & & & & 1.76047 & 0.98651 & 1.50197 \\
\hline & & & & & & 3 & & & & & 1.76047 & 0.72977 & 1.47210 \\
\hline & & & & & & 4 & & & & & 1.76047 & 0.95292 & 1.32779 \\
\hline & & & & & & 5 & & & & & 1.76047 & 1.14820 & 1.19755 \\
\hline & & & & & & & 0.5 & & & & 1.76047 & 0.92794 & 1.49264 \\
\hline & & & & & & & 1 & & & & 1.76047 & 0.99473 & 1.05682 \\
\hline & & & & & & & 1.5 & & & & 1.76047 & 1.07968 & 0.52306 \\
\hline & & & & & & & & 1.2 & & & 1.76047 & 0.95292 & 1.32779 \\
\hline & & & & & & & & 1.4 & & & 1.76047 & 0.99068 & 1.44810 \\
\hline & & & & & & & & 1.6 & & & 1.76047 & 1.01863 & 1.57780 \\
\hline & & & & & & & & & 2 & & 1.83713 & 0.97216 & 1.36543 \\
\hline & & & & & & & & & 3 & & 1.76047 & 0.95292 & 1.32779 \\
\hline & & & & & & & & & 4 & & 1.72146 & 0.94184 & 1.30620 \\
\hline & & & & & & & & & & 0.2 & 1.76047 & 0.80275 & 0.95173 \\
\hline & & & & & & & & & & 0.4 & 1.76047 & 0.94057 & 1.27674 \\
\hline & & & & & & & & & & 0.6 & 1.76047 & 0.98651 & 1.50197 \\
\hline
\end{tabular}

Table 2: Comparison of $-\theta^{\prime}(0)$ for different values of Prandtl number $\operatorname{Pr}$ when $M=0, \theta_{w}=1, D f=0, \lambda=0, E c=0$ and $R=0$.

\begin{tabular}{ccc}
\hline Pr & Chen [31] & Present result \\
\hline 1.0 & -0.58199 & -0.58223 \\
3.0 & -1.16523 & -1.16522 \\
5.0 & - & -1.56803 \\
10.0 & -2.30796 & -2.30798 \\
100.0 & - & -7.76564 \\
\hline
\end{tabular}

- $\quad$ The velocity profile and momentum boundary layer thickness decays for enhancing values of $\beta$ and $M$.

- $\quad$ The mass transfer rate scale back for higher values of $S c$.

- $\quad$ For the extend values of $S r$ increases the concentration profile and its associated boundary layer thickness.

- $\quad$ Rate of heat transfer reduces for enhancing values of Pr.

- $\quad$ Temperature profile and thermal boundary layer thickness increases by increasing values of $E c$. 


\section{Nomenclature}

$B_{0}$ magnetic field strength

$C$ fluid concentration

$C_{f}$ local skin friction coefficient

$D$ mass diffusivity

Df Dufour number

Ec Eckert number

$f$ dimensionless stream function

$g$ acceleration due to gravity

$G r_{x}, G r_{x}^{\star}$ thermal and solutal Grashof numbers

$k$ thermal conductivity

$k^{\star}$ mean absorption coefficient

$M$ magnetic parameter

$N$ buoyancy force parameter

$N u_{x}$ Local Nusselt number

Pr Prandtl number,

$R e_{x}$ local Reynolds number based on $x$,

$R$ radiation parameter

Sc Schmidt number

$S r$ Soret number

$S h_{x}$ local Sherwood number

$T$ fluid temperature

$u_{w}$ velocity of the stretching surface

$x, y$ Cartesian coordinates

$u, v$ velocity components in the $x$ - and $y$-directions, respectively

\section{Greek symbols}

$\alpha$ thermal diffusivity

$\beta$ Casson parameter

$\beta^{\star}$ volumetric coefficient of thermal expansion

$\beta^{\star \star}$ volumetric coefficient of expansion for concentration

$\eta$ similarity variable

$\theta$ dimensionless temperature variable

$\theta_{w}$ temperature ratio parameter

$\lambda$ Mixed convection parameter

$\sigma^{\star}$ Stefan-Boltzmann constant

$\mu$ dynamic viscosity

$v$ kinematic viscosity

$\phi$ dimensionless concentration

$\psi$ dimensional stream function

\section{Subscripts}

$\infty$ infinity

$w$ sheet surface

\section{References}

[1] N. Casson, A flow equation for pigment oil suspensions of the printing ink type. In: Rheology of disperse systems, Mill CC (Ed.) Pergamon Press, Oxford, (1959) 84-102.
[2] S.A. Shehzad, T. Hayat and A. Alsaedi, Three dimensional MHD flow of Casson fluid in porous medium with heat generation, Journal of Applied Fluid Mechanics, 9(1) (2016) 215-223.

[3] M.I. Khan, M. Waqas, T. Hayat and A. Alsaedi, A comparative study of Casson fluid with homogeneous-heterogeneous reactions, Journal of Colloid and Interface Science, 498 (2017) 85-90.

[4] G.K. Ramesh, K.G. Kumar, S.A. Shehzad and B.J. Gireesha, Enhancement of radiation on hydromagnetic Casson fluid flow towards a stretched cylinder with suspension of liquid-particles, Canadian Journal of Physics, 999 (2017) 1-7.

[5] G.S. Seth, R. Tripathi and Mishra, Hydromagnetic thin film flow of a Casson fluid in a non-Darcy porous medium with Joule dissipation and Navier's partial slip, Appl. Math. Mech. (English Edition), 38 (11) (2017) 1613-1626.

[6] G.T. Thammanna, K.G. Kumar, B.J. Gireesha and G.K. Ramesh, Three dimensional MHD flow of couple stress Casson fluid past an unsteady stretching surface with chemical reaction, Results in physics, 7 (2017) 4104-4110.

[7] K Ganesh Kumar, BJ Gireesha, BC Prasannakumara, GK Ramesh, Phenomenon of radiation and viscous dissipation on Casson nanoliquid flow past a moving melting surface, Diffusion Foundations, 11 (2017) 33-42.

[8] K.G. Kumar, B.J. Gireesha, M.R. Krishnamurthy and B.C. Prasannakumara, Impact of convective condition on marangoni convection flow and heat transfer in Casson nanofluid with uniform heat source sink, Journal of Nanofluids, 7 (1) (2018) 108-114.

[9] K Ganesh Kumar, B.J. Gireesha, B.C. Prasannakumara and O.D. Makinde, Impact of chemical reaction on marangoni boundary layer flow of a Casson nano liquid in the presence of uniform heat source sink, Diffusion Foundations, 11 (2017) 22-32.

[10] C.H. Chen, Combined effects of Joule heating and viscous dissipation on magnetohydrodynamic flow past a permeable, stretching surface with free convection and radiative heat transfer, Journal of Heat Transfer, 132(6) (2010) 064503.

[11] R. Chakraborty, R. Dey, and S. Chakraborty, Thermal characteristics of EMHD flows in narrow channels with viscous dissipation and Joule heating under constant wall heat flux, Int. J. of Heat and Mass Transfer, 67 (2013) 1151-1162.

[12] S. Das, R.N. Jana and O.D. Makinde, Magnetohydrodynamic mixed convective slip flow over an inclined porous plate with viscous dissipation and Joule heating, Alexandria Engineering Journal 54(2) (2015) 251-261.

[13] K.G. Kumar, GK Ramesh, BJ Gireesha, RSR Gorla, Characteristics of Joule heating and viscous dissipation on three-dimensional flow of Oldroyd B nanofluid with thermal radiation, Alexandria Engineering Journal, https://doi.org/10.1016/j.aej.2017.06.006, (2018).

[14] K.G. Kumar, BJ Gireesha, S Manjunatha, Scrutinization of Joule Heating and Viscous Dissipation on MHD Flow and Melting Heat Transfer Over a Stretching Sheet, Int. J. of App. Mech. and Eng. 23 (2) (2018) 429-433.

[15] M. Sagheer, M. Bilal, S. Hussain, and R.N. Ahmed, Thermally radiative rotating magneto-nanofluid flow over an exponential sheet with heat generation and viscous dissipation: A comparative study, Com. in Theo. Phy., 69(3) (2018) 317.

[16] T. Muhammad, T. Hayat, S.A. Shehzad and A. Alsaedi, Viscous dissipation and Joule heating effects in MHD 3D flow with heat and mass fluxes, Results in Physics, 8 (2018) 365-371. 
[17] M. Mustafa, A. Mushtaq, T. Hayat, B. Ahmad, Nonlinear radiation heat transfer effects in the natural convective boundary layer flow of nanofluid past a vertical plate: A numerical study, PLOS ONE, 9(9) (2014) e103946 1-10.

[18] O.D. Makinde, I.L. Animasaun, Thermophoresis and Brownian motion effects on MHD bioconvection of nanofluid with nonlinear thermal radiation and quartic chemical reaction past an upper horizontal surface of a paraboloid of revolution, Journal of Molecular Liquids, 221 (2016) 733-743.

[19] S.T. Mohyud-Din, S.I. Khan, Nonlinear radiation effects on squeezing flow of a Casson fluid between parallel disks, Aerospace Science and Technology, 48 (2016) 186-192.

[20] S. Rana, R. Mehmood, P.V.S. Narayana, N.S. Akbar, Free convective nonaligned non-Newtonian flow with nonlinear thermal radiation, Communications in Theoretical Physics, 66(6) (2016) 687.

[21] O.D. Makinde, K.G. Kumar, .S Manjunatha and B.J. Gireesha, Effect of nonlinear thermal radiation on MHD boundary layer flow and melting heat transfer of micro-polar fluid over a stretching surface, Defect and Diffusion Forum 378 (2017) 125-136.

[22] K.G. Kumar, B.J. Gireesha, G.K. Ramesh, N.G. Rudraswamy, Double-Diffusive Free Convective Flow of Maxwell Nanofluid Past a Stretching Sheet with Nonlinear Thermal Radiation, Journal of Nanofluids 7 (3) (2018) 499-508.

[23] C.Y. Cheng, Soret and Dufour effects on free convection boundary layer over a vertical cylinder in a saturated porous medium, Int. Com. Heat Mass Transfer, 37 (2010) 796-800.

[24] K. Bhattacharyya, G.C Layek and G.S. Seth, Soret and Dufour effects on convective heat and mass transfer in stagnation point flow towards a shrinking surface, Physica Scripta, 89 (9), (2014) Article ID- 095203.
[25] G.S. Seth, B. Kumbhakar and S. Sarkar, Soret and Hall effects on unsteady MHD free convection flow of radiating and chemically reactive fluid past a moving vertical plate with ramped temperature in rotating system, Int. J. Eng. Sci. and Tech., 7 (2) (2015) 94-108.

[26] H.R. Kataria, H.R. Patel, Soret and heat generation effects on MHD Casson fluid flow past an oscillating vertical plate embedded through porous medium, Alexandria Engineering Journal, 55(3) (2016) 2125-2137.

[27] G.S. Seth, R. Sharma, M.K. Mishra, A.J. Chamkha, Analysis of hydromagnetic natural convection radiative flow of a viscoelastic nanofluid over a stretching sheet with Soret and Dufour effects, Eng. Comp., 34 (2) (2017) 603-628.

[28] G.S. Seth, R. Tripathi and M.M. Rashidi, Hydromagnetic natural convection flow in a non-Darcy medium with Soret and Dufour effects past an inclined stretching sheet, J. Porous Media, 20 (10) (2017) 941-960.

[29] A. Kumar, R. Singh, G.S. Seth and R. Tripathi, Double diffusive MHD natural convection flow of Brinkman type nanofluid with diffusion thermo and chemical reaction effects, J. Nanofluids, 7 (2) (2018) 338-349.

[30] K.G. Kumar, M. Archana, B.J. Gireesha and M.R. Krishanamurthy, Cross diffusion effect on MHD mixed convection flow of nonlinear radiative heat and mass transfer of Casson fluid over a vertical plate, Results in Physics, 8 (2018) 694-701.

[31] C.H. Chen, Laminar mixed convection adjacent to vertical continuously stretching sheets. Heat mass transfer, 33 (1998) 471-476. 